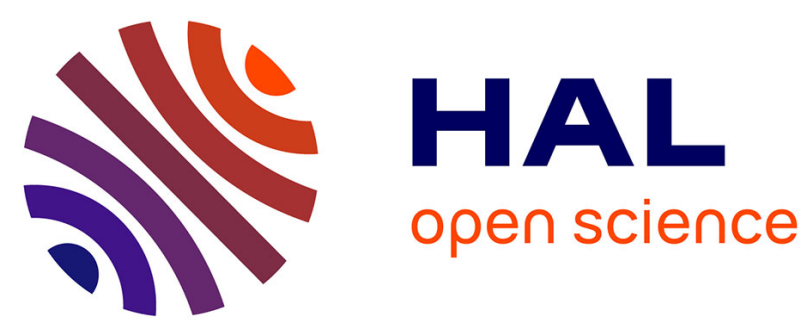

\title{
Skeletal muscle segmentation from MRI dataset using a model-based approach
}

Erwan Jolivet, Elisabeth Dion, Philippe Rouch, Guillaume Dubois, Remi

Charrier, Christine Payan, Wafa Skalli

\section{- To cite this version:}

Erwan Jolivet, Elisabeth Dion, Philippe Rouch, Guillaume Dubois, Remi Charrier, et al.. Skeletal muscle segmentation from MRI dataset using a model-based approach. Computer Methods in Biomechanics and Biomedical Engineering: Imaging \& Visualization, 2014, 2 (3), pp.138-145. 10.1080/21681163.2013.855146 . hal-03288143

\section{HAL Id: hal-03288143 \\ https://hal.science/hal-03288143}

Submitted on 16 Jul 2021

HAL is a multi-disciplinary open access archive for the deposit and dissemination of scientific research documents, whether they are published or not. The documents may come from teaching and research institutions in France or abroad, or from public or private research centers.
L'archive ouverte pluridisciplinaire HAL, est destinée au dépôt et à la diffusion de documents scientifiques de niveau recherche, publiés ou non, émanant des établissements d'enseignement et de recherche français ou étrangers, des laboratoires publics ou privés. 


\title{
Skeletal muscle segmentation from MRI dataset using a model-based approach
}

\author{
Erwan Jolivet ${ }^{\mathrm{a}}$, Elisabeth Dion ${ }^{\mathrm{b}}$, Philippe Rouch ${ }^{\mathrm{a}}$, Guillaume Dubois ${ }^{\mathrm{a}}$, Remi Charrier ${ }^{\mathrm{a}}$, Christine Payan ${ }^{\mathrm{b}}$ and Wafa Skalli $^{\mathrm{a} *}$ \\ ${ }^{a}$ Arts et Metiers ParisTech, LBM, 151 Boulevard de l'hôpital, 75013 Paris, France; ${ }^{b}$ Imagerie Médicale Hopital Louis Mourier \\ HUPNVS, Université Paris VII, INSERM U-773, Paris, France
}

\begin{abstract}
Magnetic resonance imaging (MRI) and computed tomography scans are used to assess muscle volume, but the manual segmentation, slice by slice, is long and tedious. We proposed an improvement in the deformation of a parametric-specific object method using image processing. The 3D subject-specific geometry was reconstructed based on a few selected number of MRI slices by fast rough contouring using polygons. These polygons were matched to the muscle shape by an optimisation method using an original cost function. Then, parametric-specific object was constructed and deformed. The shape was improved using a loop and the cost function in all MRI slices. The 11 main muscles of the thigh were considered, and the time required to get the shape of all muscles was $21 \mathrm{~min}$, with a volume error inferior to 5\% and a point-surface distance error (2RMS) inferior to $5 \mathrm{~mm}$. This method provides a good compromise between segmentation time and an accurate representation of the muscles shape.
\end{abstract}

Keywords: MRI; tomodensitometry; muscle; segmentation; volume

\section{Introduction}

Muscle volume decrease is an important factor related to the muscle weakness observed with age (Narici et al. 2003), neuromuscular pathology (Malaiya et al. 2007) or injury (Williams et al. 2005). In vivo muscle volume estimation is then of great interest for clinical patient follow-up as for biomechanical analysis, which requires patient-specific muscle geometry (Arnold et al. 2000; Blemker et al. 2007). However, muscle volume estimation remains a great challenge when seeking for accuracy, reproducibility and speed.

Muscle volume has been evaluated using magnetic resonance imaging (MRI) axial slices (Tracy et al. 1999, 2003; Fukunaga et al. 2001; Lund et al. 2002; Eng et al. 2007; Morse et al. 2007; Sudhoff et al. 2009) or computed tomography (CT)-scan axial slices (Rice et al. 1989; Overend et al. 1992; Jolivet et al. 2008). Muscle 3D reconstruction was assessed by manual contouring of many images in which muscle of interest was present (Tracy et al. 1999; Fukunaga et al. 2001; Eng et al. 2007), but the process is long and tedious, and such studies are only conducted on a limited number of subjects. Muscle volume was also estimated using only a given number of cross-sectional muscle area (Lund et al. 2002; Tracy et al. 2003; Morse et al. 2007), but in such cases, the muscle shape is not accessible. Very few studies use automatic muscle segmentation because of the noise included in the images. To our knowledge, only two studies focus on muscle segmentation on MRI using model-based approach applied for the first one on masseter muscles and for the second one (Gilles and Magnenat-Thalmann 2010) on the lower limb muscles. The first method implies the construction of a first coarse segmentation based on a priori knowledge of the specific muscle geometry followed by an optimisation of the muscle boundary, whereas the second method uses a scalable simplex surface equipped with reversible medial representations which could be less adapted for pathological patient.

Our group recently proposed a method, based on the deformation of a parametric-specific object (DPSO), to assess muscle geometry using a reduced number of axial images (Jolivet et al. 2008): a manual contouring is performed on this reduced set of images, then a parametric shape-based interpolation combined with a kriging technique allows to get a surface model without using the intermediate CT slices and without any a priori knowledge related to the muscle shape. The method has been validated (Jolivet et al. 2008), and it has been shown that the accuracy of muscle volume estimation using DPSO method (i.e. a function of the reduced number of slices used for muscles) converges very quickly (Sudhoff et al. 2009). Although this method provides a drastic improvement in reconstruction time, it still requires a thorough digitisation of the reduced set of slices. Moreover, from the obtained solution, image-processing techniques could allow automatic improvement by taking into account the intermediate images.

*Corresponding author. Email: wafa.skalli@ensam.eu 


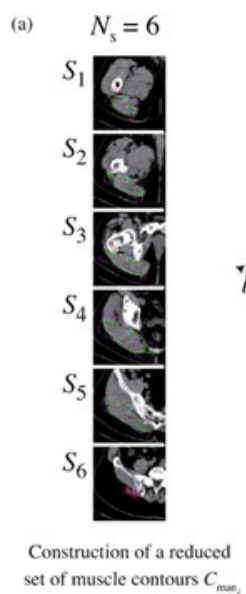

(b)

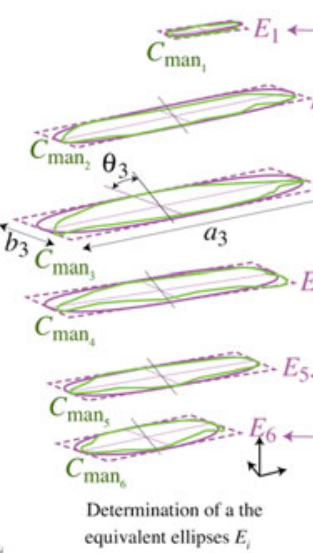

(c)

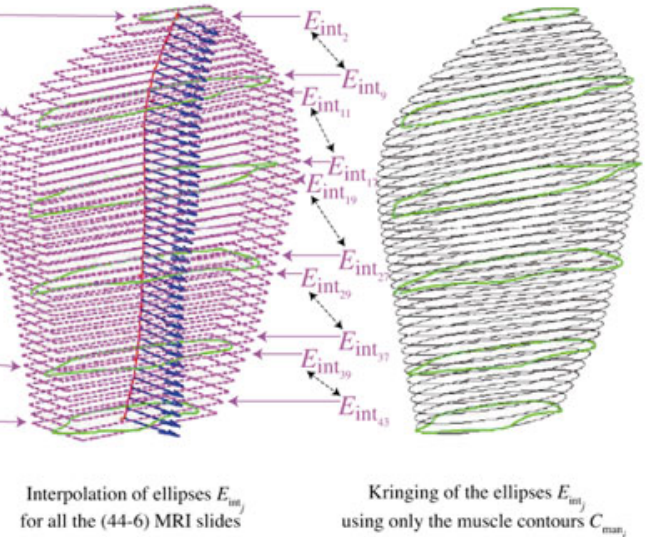

(e)

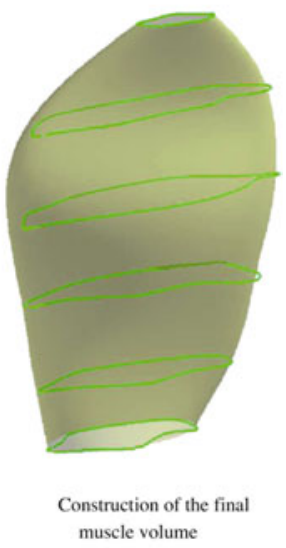

Figure 1. DPSO method presented for a reduced set of six slices.

The objective of this study was to propose and evaluate an improvement in the DPSO muscle segmentation method based on specific image analysis.

\section{Materials and methods}

The proposed method is based on the DPSO method, which is briefly described hereafter and illustrated in Figure 1.

First, from all the slices associated with a dedicated muscle, a reduced number $N_{\mathrm{s}}$ of slices are considered (Figure 1(a)): two at the extremities $\left(S_{1}\right.$ and $\left.S_{N_{\mathrm{s}}}\right)$ and the others at regularly spaced levels.

On each slice $S_{i}$ of the reduced set ( $i$ is the slice number), a fine manual contouring $C_{\operatorname{man}_{i}}$ is performed. Then, a best-fit ellipse $E_{i}$ is associated with the contour, described by three parameters: length $a_{i}$, width $b_{i}$ and orientation $\theta_{i}$ with regard to a global coordinate system (Figure 1(b)). A set of $n$ equidistant points is defined both on the contour and on the ellipse, respectively, named $P C_{i j}$ and $P E_{i j}$ ( $j$ is the point number).

For each intermediate slice ( $k$ is the intermediate slice number), which is not contoured, an intermediate ellipse $E_{\text {int }_{k}}$ is defined using cubic spline interpolation of the descriptive parameters (Figure 1(c)). $n$ equally distributed points $P E_{k j}$ are also considered.

The whole set of points on the ellipses $\left(P E_{i j}\right.$ and $\left.P E_{k j}\right)$ describe a simplified parametric model of the muscle (Figure 1(c)). The points on the contours of the reduced set $\left(P C_{i j}\right)$ are used as control points to deform this simplified model using a kriging technique (Trochu 1993), yielding the solution for muscle parametric subject-specific model (Figure 1(d)) allowing for muscle volume computation (Figure 1(e)). In this study, image processing was used to improve the process at two levels:

- Semi-automatic contouring of the reduced set of images, with a fast manual contouring followed by automatic adjustment,
- Automatic adjustment of the intermediate contours resulting from the kriging technique in order to take advantage of the information on the image and to increase the accuracy.

In both cases, automatic adjustment of an initial contour is done using an optimisation method with a specific cost criterion that is detailed hereafter.

\subsection{Simplified contour generation and its optimisation}

In this new approach, the fine manual contouring $C_{\operatorname{man}_{i}}$ that was performed on the reduced set of slices is substituted by a rough polygon $C_{\text {poly }_{i}}$ (Figure 2(a)) in order to gain segmentation time. Then, an image-based optimisation process is carried out to improve this polygonal contour automatically, leading to a robust optimised contour $C_{\mathrm{opt}_{i}}$ (Figure 2(c)).

For that, $p$ points $P C_{\text {poly }_{i j}}$ are equally distributed on the polygon contour as presented in Figure 2(b) ( $j$ is the number of the point on the contour). A cost function $G$ is then used to identify the optimal position $X_{i j k}$ of these points along the local normal of the polygonal contour in their close neighbourhood $(k$ is the number of the point along the normal). $k=2 / 3 \times p$ was arbitrarily chosen and equally distributed, respectively, along $\vec{n}$ and $-\vec{n}$ directions.

This cost function $G$ is the product of three terms: $G_{\text {Grad }}$ (image gradient), $G_{\text {Int }}$ (pixel intensity) and $G_{\text {Dist }}$ (distance to the initial contour). They are defined as follows:

- Gradient cost function $G_{\text {Grad }}\left(X_{i j k}\right)$ : a Sobel filter in the two orthogonal directions in the image yielded the gradient value $\operatorname{Grad}\left(X_{i j k}\right)$ for each point $X_{i j k}$. Then, a gradient cost was computed using a LaplacianGaussian function (Sobel and Feldman 1973):

$$
G_{\text {Grad }}\left(X_{i j k}\right)=\frac{1}{\sigma \sqrt{2 \pi}} \mathrm{e}^{-\frac{1}{2}\left(\operatorname{Grad}\left(X_{i j k}\right)-\mu\right) / \sigma}
$$



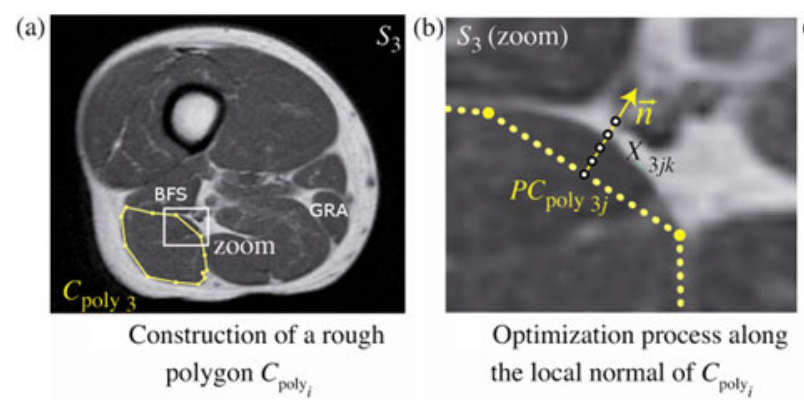

Figure 2. Optimisation process of the rough contouring.

with $\mu=\max _{k}\left(\operatorname{Grad}\left(X_{i j k}\right)\right)$ and $\sigma=\mu / 2$

This function allowed to detect the variation in pixel intensity. Specifically in the example of Figure 2, the function was used to detect the boundaries between the muscular tissue (in black) and the fat tissue (in white). The Gaussian function grants a higher importance to the high value of the gradient.

- Pixel intensity cost function $G_{\text {Int }}\left(X_{i j k}\right): R_{\text {poly }}$ and $R_{X}$ were regions defined by the contour $C_{\mathrm{poly}_{i}}$ and the modified contour $C_{\text {poly }_{i}}^{\prime}$ considering the location of the point $X_{i j k} . P($.) denotes the number of pixel in a region, mean(.) the mean intensity of pixel in a region and $\operatorname{var}($.$) the variance of pixel intensity:$

$$
\begin{aligned}
& \text { If } P\left(R_{X}\right) \geq P\left(R_{\text {poly }}\right): \\
& G_{\text {Int }}\left(X_{i j k}\right)=1-\frac{\mid \text { mean }\left(R_{X}-R_{\text {poly }}\right)-\text { mean }\left(R_{\text {poly }}\right) \mid}{2 \sqrt{\operatorname{var}\left(R_{\text {poly }}\right)}} .
\end{aligned}
$$

The added region should have the same pixel intensity as the initial region. The more the $G_{\mathrm{Int}}\left(X_{i j k}\right)$ is close to 1 , the more similar the pixel intensities are.

$$
\begin{aligned}
& \text { If } P\left(R_{X}\right)<P\left(R_{\text {poly }}\right): \\
& G_{\text {Int }}\left(X_{i j k}\right)=\frac{\mid \text { mean }\left(R_{\text {poly }}-R_{X}\right)-\operatorname{mean}\left(R_{\text {poly }}\right) \mid}{2 \sqrt{\operatorname{var}\left(R_{\text {poly }}\right)}} .
\end{aligned}
$$

The removed region should have a different pixel intensity from the initial area. In this case, the larger the $G_{\text {Int }}\left(X_{i j k}\right)$ is close to 1 , the more different the pixel intensities are.

This function allowed to choose the point among those which present a high value of $G_{\text {Grad }}$ and to keep a global coherence of the pixel intensity if no points are highlighted with the $G_{\text {Grad }}$ function.

- Distance cost function $G_{\text {Dist }}\left(X_{i j k}\right)$ :

$$
G_{\text {Dist }}\left(X_{i j k}\right)=\frac{\max _{p}\left(\left\|X_{i j p}-P C_{\text {poly }_{i j}}\right\|\right)-\left\|X_{i j k}-P C \operatorname{poly}_{i j}\right\|}{\max _{p}\left(\left\|X_{i j p}-P C_{\text {poly }_{i j}}\right\|\right)} .
$$

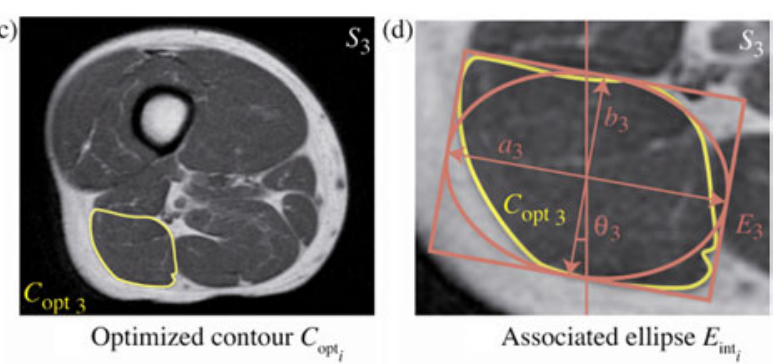

This function allowed to limit the deviation of the contour if the boundaries between two muscles were not distinct. For example, in Figure 2, the biceps femoris long head muscle (segmented muscle) and the semitendinosus muscle (on the right of the segmented muscle) were difficult to distinguish.

- Cost function definition $G$ :

$$
G\left(X_{i j k}\right)=G_{\mathrm{Int}}\left(X_{i j k}\right) \cdot G_{\mathrm{Int}}\left(X_{i j k}\right) \cdot G_{\mathrm{Dist}}\left(X_{i j k}\right) .
$$

- All the positions of $p$ points of the contour $C_{\text {poly }}$ are optimised at the minimum of the cost function $G\left(X_{i j k}\right)$, and $C_{\mathrm{poly}_{i}}$ became $C_{\mathrm{opt}_{i}}$ (Figure 2(c)):

$$
C_{\mathrm{opt}_{i}}=\left\{\max _{j, k} G\left(X_{i j k}\right)\right\}
$$

\subsubsection{Interpolation process}

The two steps of the interpolation process are derived from the classical DPSO method, briefly described in the following.

(1) For each optimised contour $C_{\mathrm{opt}_{i}}$, an equivalent ellipse $E_{i}$ is determined (Jolivet et al. 2008). This ellipse is described by its centre, axis orientations, width and length parameters as previously described (Figure 2(d)). Then, intermediate ellipses $E_{\text {int }_{j}}$ are defined on each slice that is not incorporated into the reduced set of slices. A cubic spline interpolation is used to evaluate the values of parameters for the location of these slices. On all the ellipses $E_{i}$ and $E_{\mathrm{int}_{j}}, p$ points are equally distributed in the $3 \mathrm{D}$ coordinate system related to the image acquisition device. Thus, a 3D surface object is defined, based on these 3D points of the whole set of ellipses.

(2) The second step consisted of a nonlinear deformation on this surface, using kriging algorithm (Trochu 1993; Jolivet et al. 2008). Points that described $E_{i}$ are used as control points to globally deform the $3 \mathrm{D}$ surface object so that each of those points fits exactly description points of the $C_{\mathrm{opt}_{i}}$ contours. At the end of 
this interpolation process, the first estimate of muscle contour $C_{\text {int }_{k}}$ is obtained for each intermediate slice. These contours are progressively used as an initial solution for the muscle segmentation of intermediate slices using an iterative process described in the following paragraph.

\subsubsection{Iterative process}

The iterative process is illustrated in Figure 3. The intermediate contours $C_{\text {int }_{k}}$ that are adjacent to $C_{\text {opt }_{i}}$ contours are denoted $C_{\text {adj }_{k}}$. They are optimised using the method described previously and become muscle optimised contours denoted $C_{\text {opt,adj }}$. Therefore, the reduced set of optimised contours includes both the previous $C_{\mathrm{opt}_{i}}$ and the new $C_{\mathrm{opt}_{\text {,adj }}}$ contours. The interpolation process is then applied for slices in which contours have not yet been optimised. This iterative procedure is applied until an optimised muscle contour is defined for each slice between $S_{1}$ and $S_{N_{\mathrm{s}}}$.

\subsection{Muscle segmentation evaluation}

MRI acquisitions of the hip and the thigh were acquired for four subjects (Table 1).

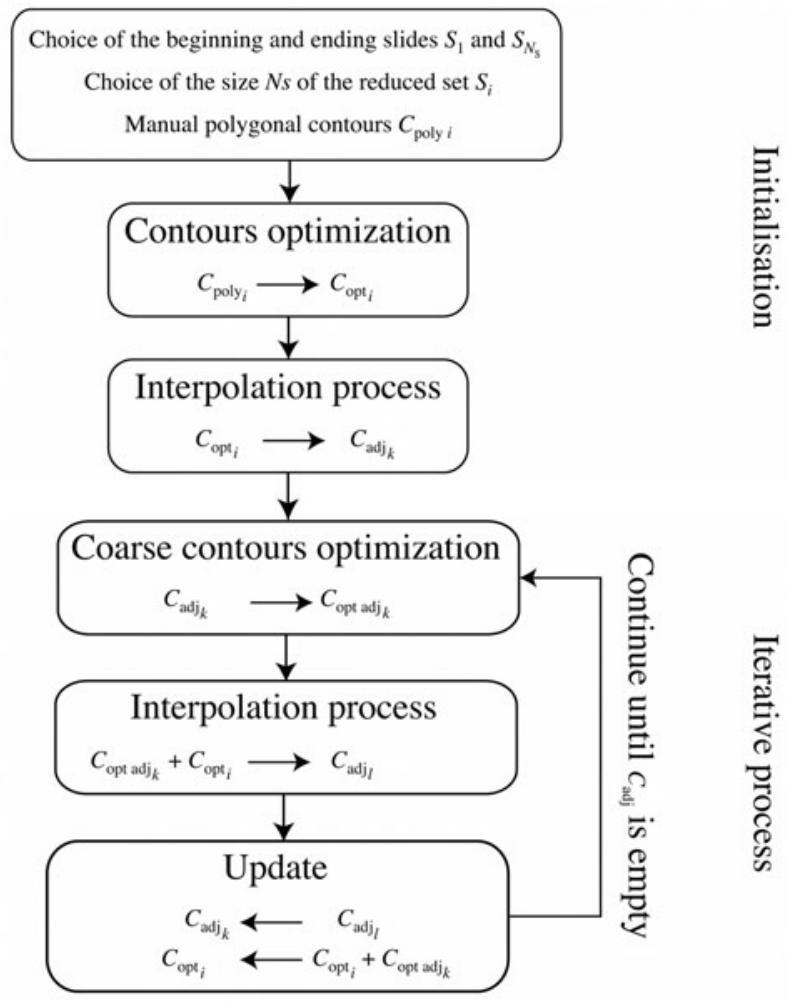

Figure 3. Illustration of muscle segmentation procedure. $C_{\text {poly }}$, rough manual polygon; $C_{\text {opt }_{i}}$, optimised contour; $C_{\text {adj }}$, adjacent to an optimised contour (before optimisation); and $C_{\mathrm{opt}, \text { adj }}$, adjacent to an optimised contour (after optimisation).
Table 1. Population characteristics: age (years), height $(\mathrm{cm})$ and weight $(\mathrm{kg})$.

\begin{tabular}{lcccc}
\hline & Sex & Age (years) & Weight $(\mathrm{kg})$ & Height $(\mathrm{cm})$ \\
\hline Subject 1 & F & 27 & 53 & 162 \\
Subject 2 & F & 32 & 81 & 181 \\
Subject 3 & F & 27 & 82 & 182 \\
Subject 4 & F & 26 & 56 & 174 \\
Mean & & 28 & 68 & 175 \\
STD & & 2.7 & 15.6 & 9.2 \\
\hline
\end{tabular}

The local ethical committee granted its approval for this study (CPP Ile de France VI, Hôpital Pitié- Salpêtriére, Paris, France, 119-08). A Philips Panorama HFO 1.5 T MRI was used to obtain a series of axial images from the superior iliac crest to the ankles in decubitus position of the subject with feet taped together. Acquisitions were realised with spin echo T1 sequence (TR $680 \mathrm{~ms}$ and TE $50 \mathrm{~ms}$ ) and the Q-body coil. Slice thickness was $10 \mathrm{~mm}$ with a pixel resolution of $0.78 \times 0.78 \mathrm{~mm}^{2}$.

Muscles of the thigh were considered. The anterior muscle group was composed of rectus femoris (RF), vastus lateralis, vastus medialis and vastus intermedialis that were grouped together in the vastus muscle group (VMG), sartorius (S) and tensor fasciae latae muscle (TF). Biceps longus (BL), biceps brevis (BB), semitendinous (ST) and semimembranous (SM) of the posterior muscle group were also reconstructed for all subjects as gracilis muscle $(\mathrm{G})$.

First, to evaluate the segmentation error induced by the automatic contour optimisation of the rough polygon, the point-to-curve distance between the points of the proposed method and the reference contour was computed. For seven slices, 50 points per slice, per muscles were projected along the normal of the reference contour, and the distance between the pair of points was calculated. The 2RMS point-to-curve distance error was studied for each muscle.

Second, muscles included in this study were manually outlined in all images of acquisition. For each muscle, using all contours, a 3D surface object was generated, named $M_{\text {Ref }}$, and reference muscle volume $\left(V_{m, \text { Ref }}\right)$ was computed for each muscle.

Finally, to analyse the sensitivity of the muscle segmentation to the size of the reduced set of MRI images $N_{S}$, the convergence of the muscle volume $V_{m, N_{\varsigma}}$ and of the 3D muscle shape $M_{N_{\mathrm{s}}}$ was evaluated with $N_{\mathrm{S}}$ varied from 2 to 7 , as seven slices are sufficient to obtain convergence (Sudhoff et al. 2009). To estimate shape differences between $M_{\text {Ref }}$ and $M_{N_{\mathrm{s}}}$, point-to-surface distance was determined between each point of $M_{N_{\mathrm{s}}}$ and surface of $M_{\text {Ref }}$. Mean distance and 2 root-mean-square (2RMS) that corresponded to $95 \%$ confidence interval were computed for each $M_{N_{\mathrm{s}}}$. 
Table 2. Number of slices for each of the nine visible muscles included in the study (mean, maximum and minimum) and reference muscle volume computed from the segmentation generated from muscle contours (mean, maximum and minimum).

\begin{tabular}{llcr}
\hline Muscle & Abbreviation & $\begin{array}{c}\text { Number of slices } \\
\text { Mean (min-max) }\end{array}$ & $\begin{array}{c}\text { Reference Volume }\left(\mathrm{cm}^{3}\right) \\
\text { Mean }(\mathrm{min}-\mathrm{max})\end{array}$ \\
\hline Biceps femoris short head & BFS & $17(14-20)$ & $91(66-121)$ \\
Biceps femoris long head & BFL & $22(17-27)$ & $159(112-203)$ \\
Rectus femoris & RF & $25(22-26)$ & $236(193-288)$ \\
Gracilis & GRA & $26(24-29)$ & $92(57-121)$ \\
Sartorius & SAR & $40(38-44)$ & $123(91-157)$ \\
Semimembranosus & SM & $13(14-19)$ & $165(97-236)$ \\
Semitendinosus & ST & $24(15-28)$ & $160(104-225)$ \\
Tensor fasciae latae & TFL & $12(10-13)$ & $59(40-83)$ \\
Vastus intermedialis & & $24(21-28)$ & $1394(1048-1774)$ \\
Vastus lateralis & VMG & & \\
Vastus medialis & & & \\
\hline
\end{tabular}

\section{Results}

The mean number of image on which each muscle was visible is indicated in Table 2. The global 2RMS point-tocurve distance error for the contour segmentation was $1.29 \mathrm{~mm}$, with a maximum of $2.26 \mathrm{~mm}$ for the BFS muscle and a minimum for GRA muscle with $0.63 \mathrm{~mm}$ (Table 3 ).

The volume difference (Figure 4) decreases when the number of manual contours $N_{\mathrm{s}}$ increases. If we consider a difference lower than $5 \%$, six manual muscle contours would be required for ST muscle, five for BFS, RF and SAR and four for BFL, GRA, SM, TFL and VMG, instead of seven for RF and SAR, six for BFL, five for BFS, GRA, SM, TFL and $\mathrm{ST}$ and four for VMG with the non-optimised method.

Point-to-surface distance between surface $M_{N_{\mathrm{s}}}$ and surface of $M_{\text {Ref }}$ (Figure 5) decreases when the number of manual contours $N s$ increases. If we consider a difference lower than $5 \mathrm{~mm}$, five manual muscle contours would be required for RF, SM, ST and VMG muscles, four for BFS, BFL, SAR and TFL and three for GRA.

\section{Discussion}

This study proposed a method to obtain a fast and accurate segmentation of muscle and was applied on the muscles of the thigh. This method required manual muscle contours on a reduced number of images, and the segmentation process was realised in two steps: the first coarse segmentation followed by an optimisation iterative procedure. With respect to the already published techniques, this new development regards the automatic contour optimisation (ACO) based on a cost function containing an original coherence criterion. ACO allowed time reduction in the manual processing of the reduced set of contours. It also allowed automatic adjustment of the intermediate slices that were previously unprocessed using DPSO method. The cost function appeared efficient because the DPSO method provided an initial solution, which was already close to the adjusted one. Table 4 summarises the improvements in the proposed method.

The sensitivity analysis of the number of manual muscle contours showed that the volume difference between the segmented muscle and the reference muscle volume, obtained by manually contouring all the images in which muscle of interest was present, lowered to $5 \%$ with only 4-6 manual muscle contours, depending on the muscle. While the previous DPSO method needed a reduced set of seven slices and 26 min to build the whole model, this new approach needs only five slices and $21 \mathrm{~min}$, which is of great interest regarding the classical manual segmentation time that was up to $80 \mathrm{~min}$. A significant part of the enhancement is due to the iterative interpolation process that is very efficient on long-curved path muscle such as the SAR.

Some limitations had to be dealt with. A difficulty was to identify slices where considered muscles appeared or disappeared because the muscle-tendon transition is not really clear. A previous study has shown that this uncertainty of these extreme slice locations leads to an error estimation lower than $2.1 \%$, depending on the muscle (Sudhoff et al. 2009). However, this requires well-trained operators, and further improvements are necessary to reduce operator dependence, particularly in longitudinal studies in which the muscles change and the operators are not necessarily the same.

Table 3. Point-to-curve distance error between the points of the proposed method and the reference contour ( 7 slices, 50 points per slice).

\begin{tabular}{lcccccccccc}
\hline Muscle & BFL & BFS & GRA & RF & SAR & ST & SM & TFL & VMG & Global \\
\hline Mean & 0.65 & 0.88 & 0.42 & 0.75 & 0.62 & 0.59 & 0.69 & 0.56 & 0.73 & 0.70 \\
2*RMS & 1.08 & 2.26 & 0.63 & 1.24 & 1.35 & 0.88 & 1.09 & 0.98 & 1,06 & 1.29 \\
\hline
\end{tabular}


BFS

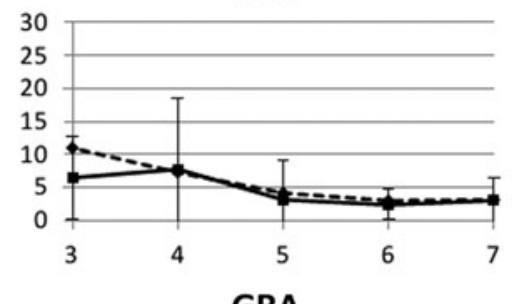

GRA

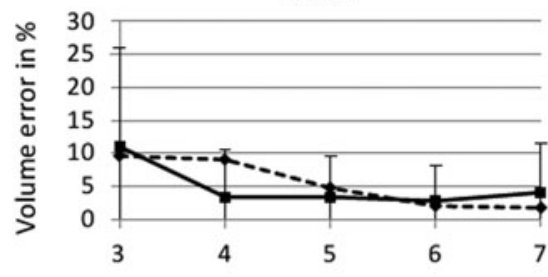

ST

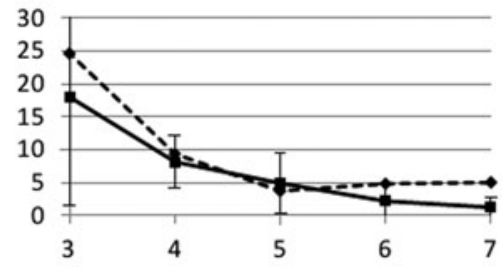

BFL

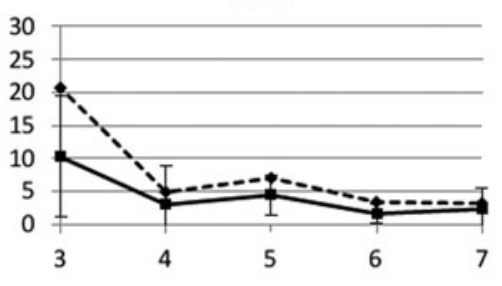

SAR

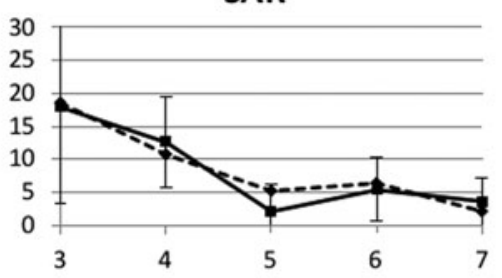

TFL

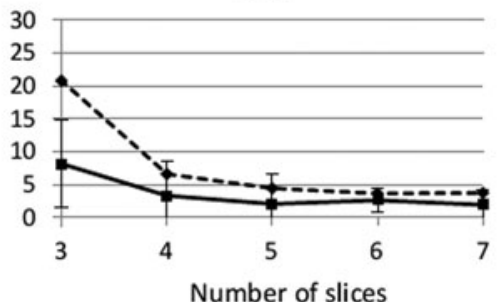

RF
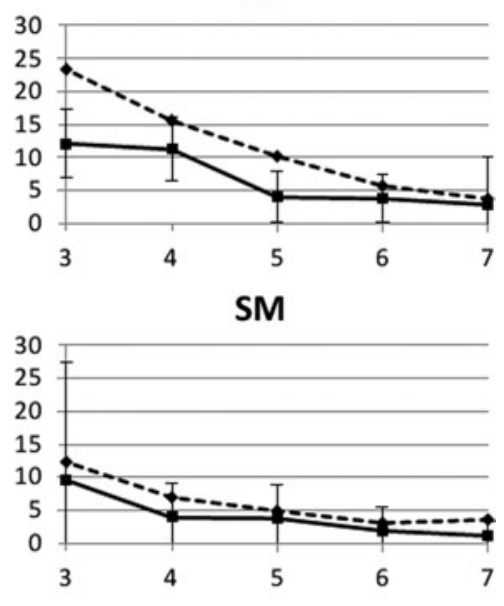

VMG

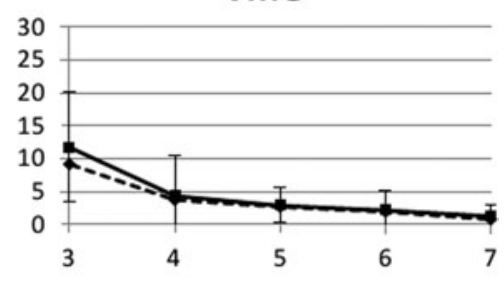

Figure 4. Volume difference in per cent between reference volume $V_{m, \text { Ref }}$ and segmented volume $V_{m, N_{\mathrm{s}}}$ for each muscle included in the study (dotted line represents the DPSO method Jolivet et al. (2008) and continuous line represents the presented method).
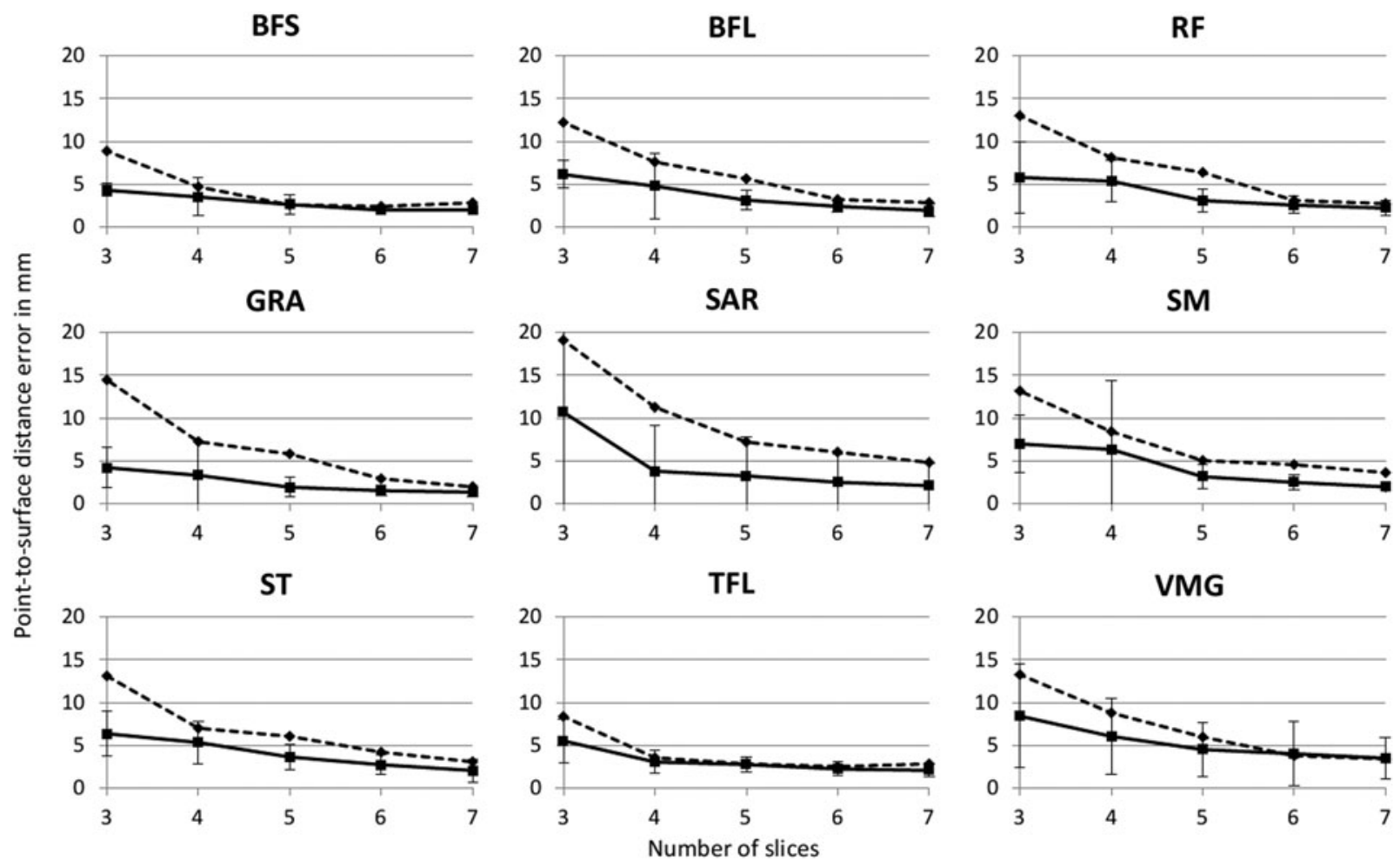

Figure 5. Point-to-surface distance between surface $M_{N_{\mathrm{s}}}$ and surface of $M_{\text {Ref }}$ for each muscle included in the study (dotted line represents the DPSO method Jolivet et al. (2008) and continuous line represents the presented method). 
Table 4. Main differences between the DPSO method and the presented method.

\begin{tabular}{lll}
\hline Automatic image processing & DPSO method & Proposed method \\
$\begin{array}{l}\text { Muscle contour segmentation in a reduced } \\
\text { set of slices }\end{array}$ & No & $\begin{array}{c}\text { Automatic contour optimisation (ACO) using an } \\
\text { original cost function } \\
\text { Rough polygon }+ \text { ACO }\end{array}$ \\
$\begin{array}{l}\text { Automatic estimation of intermediate muscle contours } \\
\begin{array}{l}\text { Automatic adjustment of the estimated intermediate } \\
\text { muscle contours }\end{array}\end{array}$ & Yes (DPSO) & Yes (DPSO) \\
\hline
\end{tabular}

As for various medical images, segmentation is an uneasy task because of the noise included in the images. Especially the boundary of two muscles (e.g. whole vastus or BFS and BFS) was not easy to identify. The cost function $G_{\text {Dist }}\left(X_{i j k}\right)$ limited the aberrant solution, especially during the iterative process. A manual correction might be necessary to improve the estimated shape. For this study, no manual corrections were made.

The location of the slices $S_{i}$ included in the reduced set could affect the definition of the first coarse segmentation; consequently, refinement step would also be affected. In this study, these slices were equally spaced. The cost function that was used for the optimisation process combined the classical gradient cost function with two functions related to coherence criteria: a distance criterion, based on the fact that the initial contour is already close to the real one, and a pixel intensity criterion, based on the fact that the global signal intensity for the muscle is clearly differentiated from the surrounding. The distance criterion can be less relevant for muscles that undergo wide and sudden change in shape and cross-sectional area, because in this case the initial contour for the optimisation process remains quite different from the real contour, and the combined criteria for segmentation did not fully succeed in retrieving this solution: particularly, the $10-\mathrm{mm}$ slice thickness used in this study, corresponding to routine clinical protocol, is a limitation, while thinner slices would probably allow to take progressively into account this shape variation. For such routine protocols, it could be interesting to use training data-set to determine the optimal location of the slices for manual contouring $(\mathrm{Ng}$ et al. 2009). Nevertheless, in general, the iterative process proposed in this study with optimisation of muscle contour gradually ensured to optimise an estimated muscle contour that was close to muscle contour solution that would be obtained with full manual reconstruction.

Some muscles were inconstantly distinguishable because the fascia between those two muscles is not visible in most of the cases. This was the case of the vastus medialis muscle and the vastus lateralis muscle, and their volume was evaluated together (Sudhoff et al. 2009).

Another limitation was the difficulty in evaluating the reference muscle volume in vivo. In many studies as in this study, the reference standard measure of muscle volume was the volume computed using manual muscle contour in all images in which muscle was present. Thus, accuracy of this reference volume estimation was not clear. Manual segmentation of in vitro MRI acquisition of forearm followed by dissection and volume measurement demonstrated that volume estimation accuracy was related to area-to-volume ratios and related error was $>10 \%$ for high value of this ratio (Eng et al. 2007). Moreover, interoperator reproducibility study, including two operators, focused on muscles crossing the knee and showed that volume error estimation between the operators with entire manual procedure on MRI acquisition of the lower limb was between $2.7 \%$ and $13.9 \%$ depending on the muscles (Sudhoff et al. 2009).

The image processing with the use of the cost function allowed an easier way to segment the muscle contours. However, this method induced a global 2RMS point-tocurve distance error of $1.29 \mathrm{~mm}$. This error was due to the difficulty in distinguishing the boundaries between the muscles, especially for the BFS muscle, which had an error of $2.26 \mathrm{~mm}$. GRA appeared as the easier muscle to segment, as it is along the fat tissue and has a thick fascia and had an error of $0.63 \mathrm{~mm}$. Those muscles are visible in Figure 2(a).

In conclusion, the method of muscle segmentation presented allowed estimating the muscle volume in vivo by minimising human intervention from MRI acquisition. Using the results of sensitivity analysis, clinicians could define the number of manual contours to perform muscle segmentation for a given expected precision on volume estimation.

\section{Acknowledgements}

The authors acknowledge Jérôme Hausselle for his help in this study. This work was partly funded by Association Française contre les myopathies (AFM, program n 14068) and by the Société Générale within the BiomecAM chair program on subject-specific musculoskeletal modelling.

\section{References}

Arnold AS, Salinas S, Asakawa DJ, Delp SL. 2000. Accuracy of muscle moment arms estimated from MRI-based musculoskeletal models of the lower extremity. Comput Aided Surg. 5(2): $108-119$. 
Blemker SS, Asakawa DS, Gold GE, Delp SL. 2007. Imagebased musculoskeletal modeling: applications, advances, and future opportunities. J Magn Reson Imaging. 25(2):441-451.

Eng CM, Abrams GD, Smallwood LR, Lieber RL, Ward SR. 2007. Muscle geometry affects accuracy of forearm volume determination by magnetic resonance imaging (MRI). J Biomech. 40(14):3261-3266.

Fukunaga T, Miyatani M, Tachi M, Kawakami MKY, Kanehisa H. 2001. Muscle volume is a major determinant of joint torque in humans. Acta Physiol Scand. 172(4):291-302.

Gilles B, Magnenat-Thalmann N. 2010. Musculoskeletal MRI segmentation using multi-resolution simplex meshes with medial representations. Med Image Anal. 14(3):291-302.

Jolivet E, Daguet E, Pomero V, Bonneau D, Laredo JD, Skalli W. 2008. Volumic patient-specific reconstruction of muscular system based on a reduced dataset of medical images. Comput Methods Biomech Biomed Eng. 11(3):281-290.

Lund H, Christensen L, Savnik A, Boesen J, Danneskiold-Samse B, Bliddal H. 2002. Volume estimation of extensor muscles of the lower leg based on MR imaging. Eur Radiol. 12(12):2982-2987.

Malaiya R, McNee AE, Fry NR, Eve LC, Gough M, Shortland AP. 2007. The morphology of the medial gastrocnemius in typically developing children and children with spastic hemiplegic cerebral palsy. J Electromyogr Kinesiol. 17(6):657-663.

Morse CI, Degens H, Jones DA. 2007. The validity of estimating quadriceps volume from single MRI cross-sections in young men. Eur J Appl Physiol. 100(3):267-274.

Narici MV, Maganaris CN, Reeves ND, Capodaglio P. 2003. Effect of aging on human muscle architecture. J Appl Physiol. 95(6):2229-2234.
Ng H, Ong S, Liu J, Huang S, Foong K, Goh P, Nowinski W. 2009. 3D segmentation and quantification of a masticatory muscle from MR data using patient-specific models and matching distributions. J Digit Imaging. 22(5):449-462.

Overend TJ, Cunningham DA, Paterson DH, Lefcoe MS. 1992. Thigh composition in young and elderly men determined by computed tomography. Clin Physiol. 12(6):629-640.

Rice CL, Cunningham DA, Paterson DH, Lefcoe MS. 1989. Arm and leg composition determined by computed tomography in young and elderly men. Clin Physiol. 9(3):207-220.

Sobel I, Feldman G. 1973. A $3 \times 3$ isotropic gradient operator for image processing. Pattern Classic Scene Anal. 73:271-272.

Sudhoff I, de Guise JA, Nordez A, Jolivet E, Bonneau D, Khoury V, Skalli W. 2009. 3D-patient-specific geometry of the muscles involved in knee motion from selected MRI images. Med Biol Eng Comput. 47(6):579-587.

Tracy BL, Ivey FM, Hurlbut D, Martel GF, Lemmer JT, Siegel EL, Metter EJ, Fozard JL, Fleg JL, Hurley BF. 1999. Muscle quality. II. Effects of strength training in 65- to 75-yr-old men and women. J Appl Physiol. 86(1):195-201.

Tracy BL, Ivey FM, Metter EJ, Siegel JLFEL, Hurley BF. 2003. A more efficient magnetic resonance imaging-based strategy for measuring quadriceps muscle volume. Med Sci Sports Exerc. 35(3):425-433.

Trochu F. 1993. A contouring program based on dual kriging interpolation. Eng Comput. 9:160-177.

Williams GN, Snyder-Mackler L, Barrance PJ, Buchanan TS. 2005. Quadriceps femoris muscle morphology and function after ACL injury: a differential response in copers versus non-copers. J Biomech. 38(4):685-693. 